\title{
DESIGN AND ANALYSIS OF A FLEXIBLE MANUFACTURING SYSTEM WITH SIMULATION - EFFECTS OF FLEXIBILITY ON FMS PERFORMANCE
}

\author{
Susumu Morito \\ Tomohiro Takano \\ Hisanobu Mizukawa \\ Department of Industrial Engineering and Management \\ School of Science and Engineering \\ Waseda University \\ Shinjuku, Tokyo 169
}

\author{
Kiyohisa Mizoguchi \\ Engineering Department \\ Yamazaki Mazak Corporation \\ Oguchi-cho, Aichi, 480-01
}

\section{ABSTRACT}

An extensive simulation model is developed to analyze Mazatrol FMS systems manufactured by Yamazaki Mazak, Inc. The Mazatrol FMS is a moduletype FMS consisting of several machining centers, load/unload stations, a stacker crane, pallet stocker, among others. Outline of the simulated system is described to clarify its boundary and the level of details with emphasis on its control mechanisms and parts loading rules.

The paper discusses the application of the developed simulation model to analyze the Mazatrol system. Specifically, we present an analysis to study the effects of various types of flexibility on FMS performance. In particular, the effects are studied of such factors as 1) the number of alternative machines that can perform a particular operation (which we call RFLEX), and 2) the number of pallets for each type of operation, on the system performance as measured by makespan. Our main finding, among others, is the fact that the flexibility of the overall system depends very much on RFLEX as defined above. When RFLEX $=1$ (i.e., one-to-one correspondence between an operation and a machine as in a standard job shop model), the system has limited flexibility, and flexibility increases substantially when RFLEX is increased from 1 to 2, whereas further increase in RFLEX contributes only marginally to flexibility. Implications of the simulation study on system design, control and scheduling of the FMS will be discussed.

\section{INTRODUCTION}

Flexible Manufacturing Systems (FMS) are increasingly popular in many areas of manufacturing. Installation of an FMS normally leads to substantial investment, and thus careful analysis would be necessary before making definite commitment. At the same time, FMSs generally consists of many interre- lating components and thus it, is rather difficult, to estimate their performance. Discrete event simulation provides a perfect tool to study and analyze FMSs so that users of FMSs can come up with economically sound investment and their proper operation, and that designers of FMSs can develop responsive and efficient systems.

There exist many papers on applications of simulation to analyze FMSs during the last decade. There also exist books devoted to the topic. See, e.g., Carrie(1988), Talavage and Hannam(1988). Despite the existence of many studies in the field, it is rather rare to find research which is based on a real system and its real data.

In this paper, real-life commercial FMSs manufactured by Yamazaki Mazak is considered, and a detailed simulation model of their Mazatrol FMSs is described which are developed to explore the potential of simulation as a tool to determine a particular configuration of FMS to be introduced, and also as a tool to achieve efficient operations of the system. In particular, we try to find general guidelines that lead to efficient operations of module-type Nazatrol FMSs. We focus, among various factors related to FMSs, on flexibility generated by allowance in choosing a machine to process an operation, the number of machines and of pallets, and scheduling priority, and clarify how these factors affect performance of FMSs so that the identified characteristics could be exploited to come up with an efficient system operations.

Sethi and Sethi (1990) provide an extensive survey on flexibility in manufacturing. We show through discrete event simulation how flexibility of FMSs can be achived by providing a few alternative machines for operations. The particular type of flexibility studied in this paper is "routing flexibility" according to the classification of Sethi and Sethi (1990). 


\section{DESCRIPTION OF MAZATROL FMS AND ITS MODELING}

\subsection{Components of Mazat.rol FMS}

The Mazatrol FMS, which is to be studied in this paper, is a module-type FMS consisting of machining centers, load/unload stations, a stacker crane robot, pallet stocker, FMS computer among others. The basic components of the system will be described briefly below, and the boundary of modeling effort will be clarified later.

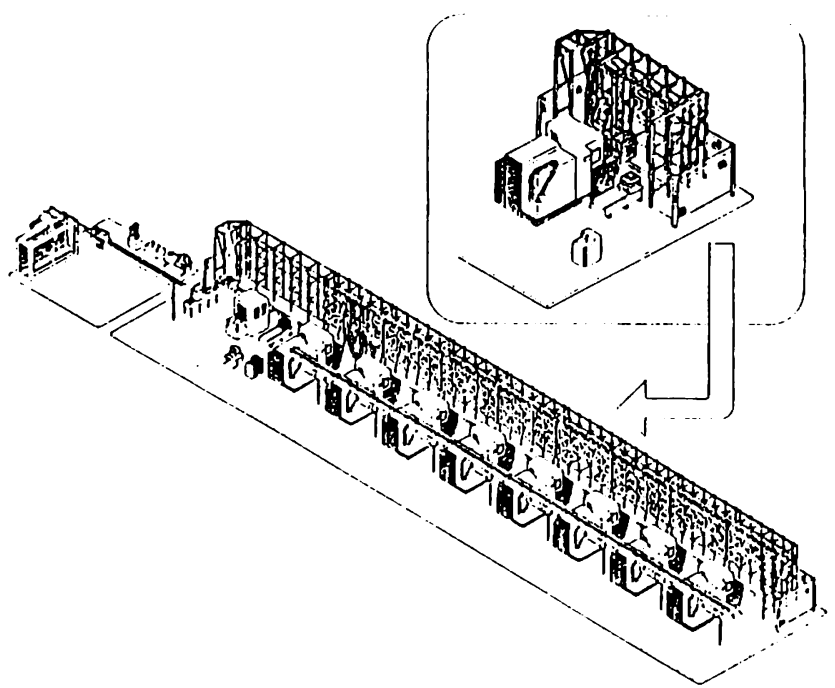

Figure 1: Mazatrol FMS

First important sub-systems of the Mazatrol FMS will be briefly described. Fig.1 shows how the Mazatrol FMS looks like.

Horizontal Machining Centers: The base machine tools of the Mazatrol FMS are highspeed, high-precision horizontal machining centers. They include a total of eight models with pallet sizes ranging from $400 \mathrm{~mm}$ to $1250 \mathrm{~mm}$.

Load/Unload St.ations: Workpiece set-up and unloading to/from pallets are conveniently performed at the load/unload stations which can be indexed every 90 degree for operator access to all sides.

Stacker Crane Robot: Pallets are transported between the loading station, the pallet stocker and the machine tools by the large scale transporteer robot. Maximum weight capacity is 8 tons.

Pallet Stocker: The stocker is used to temporarily store pallets with loaded workpieces. By having the appropriate number of prepared pallets stored in the pallet, stocker, unmanned operation can be carried out over extended periods of time. The stockers are of two or three level design for space efficiency.

FMS Computer: This computer, linked to each machine tool's CNC unit, transfers NC: programs as required and controls overall system operation. It commands the stacker crane robot to transport pallets to each machine according to the production schedule.

Tool Management System: In order to carry ont the automatic machining of a wide variety of work-pieces, a large nu mber of tools are recunired. To ensure high accuracy, a large amount, of tool data such as tool length, tool diameter, and accumulated tool usage time are managed by the Mazak tool management system. The system features read/write chips that are monnted in each tool, and data are read or written every time a tool is loaded or unloaded to/from a machining center by a non-contact sensor.

Tool Transport System: The tool transport system is comprised of a central tool hive stocker, a tool transportation robot which transports tools to/from the central tool hive stocker to/from each machining center and the tool management, computer which controls the entire system.

When a tool reaches the end of its service life on a machining center, the individual CNC unit notifies the tool management, computer which in turn supplies a new tool.

When a new workpiece is loaded on a machining center, the FMS computer sends the work number to the tool management, computer which makes a list of the tools required to machine the workpiece. The tool management computer checks the tools currently stored in the machining center's tool magazine witl the list of required tools. Tools that are not in the tool magazine are carried from the central tool hive stocker by the tool transportation robot.

The fundamental element of the Mazatrol FNIS is naturally a set of $\mathrm{m}$ horizontal machining centers (1 $\leq m \leq 8$ ), all of which have essentially identical functions, except that, each machine has distinct machining capabilities because of different sets of tools krpt, in its tool magazine. That, is, all machines can perform any operation provided that the proper set of tools are prepared for the operation. The fact that, the Mazatrol FMS consists of several functionally identical machines leads to a certain form of flexibility, which is the main theme of this study. 
The Mazatrol FMS is custom-made in the sense that its customer (or user) determines the number of machining centers $\mathrm{m}$ (simply called machines hereafter) so that the system suits for his objective, with the restriction that the number of machines is at most 8. Similarly, the number of load/unload stations can vary between 1 and 4 . Some of the load/unload stations could have washing capabilities. The system always has one stacker crane robot. The number of pallet stockers goes up to approximately 120 , and a customer again decides how many stockers in the system. Only one type of pallets exists in the system, and each pallet has its home-position on pallet stocker. As fixtures are normally placed on pallets to hold work-pieces and will not be changed too often, pallets in this paper refer to pallets on which particular fixture is already placed. In this context, the number of pallets (with identical fixturing) that can perform a particular operation is normally rather small, and mostly in the lower side of a single-digit number. An operation here actually consists of many sub-operations in most cases, and many tools are normally used to do these sub-operations. Anyway, an operation is a series of sub-operations which can be performed without changing setup. Setups normally require pallet changes.

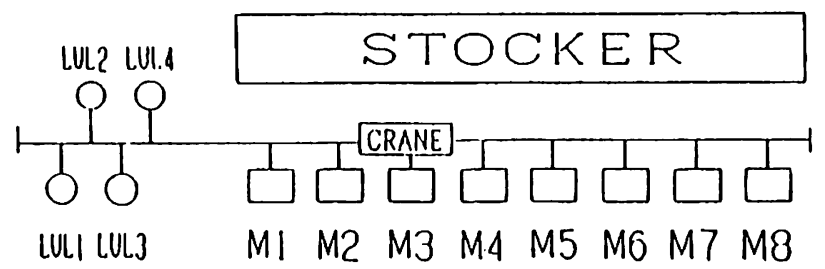

Figure 2: Element of Mazatrol FMS

Work-pieces, which we now call parts, are fixed on pallets (with a particular fixture) at load/ur.load stations (LUL), and put into the system. The stacker crane transports the parts (on a pallet) to one of the machines. When the required machining is completed, the pallet is transported back to LUL, where the parts are removed from the pallet. If there remains another operation, the parts would later be placed on a pallet for the next operation. All the movement of pallets will be performed by the stacker crane which will be controlled automatically by the FMS computer. If either machines or LUL are occupied and unavailable, pallets could wait at its home position on the stocker, which act as a common buffer of the system.

Note that human involvement of the system is limited only to the load/unload operation done at LUL.
The rest of the system is completely computer controlled.

Fig.2 shows schematically those areas of the Mazatrol FMS included in the current version of the simulation model. Specifically, note that the tool management/transport system, which is an option a user may/may not choose, is not included in the current. modeling effort.

\subsection{Fixing Variable Elements of the System for Analysis}

Since real FMSs are analyzed here, there exist, many factors which are variable and could be changed. For example, all factors which FMS users determine such as the numbers of machines, pallets, LULs, workers and stockers can all be changed as the system is modularized. Other variable factors include scheduling rule, crane control mech anism, parts processing time, tool availability, among others.

When one fixes factors and conditions that define system environment, one has to be careful so that

1) those fixed factors and conditions do not provide system bottleneck, and

2) they are fixed to technologically reasonable conditions.

If some of the fixed factors yield bottleneck, it would become difficult to see if the observed system performance reflects the effects of factors which are changed in the experiments, or is "biased" by those fixed factors that produce bottleneck. If factors are fixed unreasonably or unrealistic, the meaning of using real FMSs for analysis would be lost.

Based on the above observations, the factors are fixed as follows:

a) Use 4 LULs, which is the maximum number allowed for this FMS. Assume that all these LUIs can perform loading/unloading of any operations.

b) Time required for load/unload is constant $5 \mathrm{~min}$. irrespective of parts and operation type. More specifically, load time is 5 min. and unload time is also 5 min. Crane transportation time is assumed to be constant $1 \mathrm{~min}$. without regard to the travel distance.

c) For each operation of parts, there exist a few sets of unique pallets (with specific fixtures). Normally, there exists a one-to-one relationship between the type of pallet and a particular operation. In most cases, pallet change wonld be necessary after the completion of one operation.

d) Four workers are assumed to exist at, LULs. Namely, there exist as many workers as the number of LULs so that workers would not be a constraining resource. 
e) Simulation will be started from an empty-andidle condition where all machines, LULs, workers and crane are available. A predetermined number of raw materials are assumed to exist around the LUL area.

f) No machines, LULs, the crane are assumed to go down throughout the simulation period.

g) The real control logic of the Mazat,rol stacker crane is simulated, and the priority dispatching rule of the crane is essentially that of the Mazatrol FMS.

h) The parts loading rule used in simulation also simulates the real loading rule of the Mazatrol FMS. The loading rule and the crane control logic are integrated together, and the FMS computer essentially directs workers what to do (say, unload a part from a pallet, or load a part on a pallet).

i) The priority ordering of parts plays an essential role in the crane control logic and also in the parts loading rule. An FMS user specifies priority ordering of parts, and, for each part, specifies whether earlier/latter operations get higher priority. These specifications determine the priority ordering of all operations needed for production of all parts. The FMS computer determines the next step based on the priority ordering of operations as well as on the availability of "raw" material of operations and of pallets.

Input data with which simulation experiments are performed were generated by a technical staff of Mazak as "typical" load data of the Mazatrol FMS after carefully studying historical performance data of real systems. The data consists of 26 distinct types of parts with the total of 38 different operations. The number of operations for each part ranges from 1 to 3. The required quantity of each part is assumed to be 10 , and raw materials of the first operation of each part are assumed to be available. In total, the simulated system would produce the total of $26 \times 10$ $=260$ parts. Higher priority is given to those parts with more operations, and also to those operations with shorter processing time except for those experiments to analyze scheduling priority, where priority is randomly clinged.

\section{SIMULATION MODEL, ANALYSIS, AND EXPERIMENTS}

\subsection{Major Areas of Analysis}

To study effects of providing multiple machines which can process a particular operation, we define a measure called RFLEX as follows:

RFLEX = the number of alternative machines which can process a particular operation.

Here RFLEX refers to "routing flexibility" (See, e.g., Sethi and Sethi (1990)). The higher the RFI,EX is, the more flexible the system is expected to be. Generally, values of RFLEX would be different, for different operations. For example, operation 1 can be performed on machines $B$ and $E(R F L F X=2)$, whereas operation 2 may have to be processed only on machine A (RFLEX=1). The following four areas are studied in detail.

As mentioned earlier, the original design philosophy of the Mazatrol FMS is to prepare several funtionally identical machines. Therefore, theoretically RFLEX of the FMS is equal to $\mathrm{m}$, where $\mathrm{m}$ stands for the number of machines. However, due to nonavailability of tools, and also due to requirements on precision of parts, it may not be feasible to achieve RFLEX $=m$, nor it may be necessary to have this level of ronting flexibility.

1) system flexibility due to the existence of alternative machines

If the system has several alternative machines which can process a particular operation, routing flexibility results. Relationslip between routing flexibility and FMS performance is studied by changing RFLEX from 1 to 8 (= the number of machines). Here, we assume in our experiments that the number of alternative machines is identical for all operations. In reality, RFLEX is expected to be different for different parts.

2) effects of the number of system entities on FMS performance

Each operation of each part corresponds to a particular pallet in this system as described earlier. Parts cannot go in to the system without pallets, and thus the number of pallets essentially controls the number of entities in the system.

3) effects of the number of machines on FNIS performance and their relationship to RFLEX

The number of machines certainly is one of the most important factors that affects FMS performance. Its interrelationship with RFLEX is also studied.

4) effects of machine assignment, and scheduling priority

Actual assignment of RFIJEX candidate marhines is expected to affect the makespan performance. This would be true especially when RFLEX is smaller. Similarly, it would be of interest to study the effects of scheduling priority as determined mainly by the priority of parts.

\subsection{Performance Measure}

Since the number of parts to be produced is fixed, the makespan that is the time when the last item gets out of the system after unloading, is used as the perfor- 
mance measure. Note that the total processing time of all parts is constant, and thus the average machine utilization is the total processing time divided by $(\mathrm{m}$ $x$ the makespan), where $m$ is the number of machines.

\subsection{Experiments}

A Mazatrol simulation model was developed in SLAM II. Fortran inserts are used extensively to simulate overall Mazatrol control logic including crane control.

When there is a one-to-k correspondence between an operation and machines, and thus there exist $k$ al ternative machines for each operation, i.e., RFLEX $=k$, RFLEX alternative machines are randomly assigned to each operation. Since the system performance depends on this machine assignment especially when RFLEX is smaller, simulation would be repeated with 50 randomly generated machine assignmelt. Note that machine assignment really specifies candidate machines on which a particular operation can be processed, and does not specify a particnlar machine on which an operation is actually processed (unless RFLEX is 1 , in which case the machine is uniquely determined). Once the assignment of alternative machines is given, the rest of simulation is completely deterministic. The actual machine where machining is performed is determined by the Mazatrol control logic (based on the "current" status of the system) as simulated in the simulation model. The makespans fluctuate depending on the candidate machine assignment, and thus the results given below show the average makespan together with the associated standard deviation.

\section{RESULTS AND OBSERVATIONS}

\subsection{Effects of Changing RFLEX (Fig.3)}

As shown in Fig.3, the makespan decreases as RFI,EX get.s bigger. More specifically, the reduction of makespan is most remarkable when RFLEX is increased from 1 to 2 . Meaningful improvement of makespan, however, cannot be expected when RFLEX is raised above 2 . The graph appears to indicate microscopic increase in the makespan as RFIEX exceeds 6. It is unclear if there is any reason for the increase of makespan when there exists too much flexibility. Anyway, it is counter-intuitive if the makespan increases when the system has too much flexibility.

With regard to variability of makespans when machine assign ments have been (randomly) clianged, the variability of makespans when RFLEX is 1 is substantial, whereas the variability becomes small quickly as RFLEX is increased. Note that the variability is measured by the standard deviation of makespans obtained for various random machine assignments. It is interesting to note that the variability is almost non-existent for RFLEX greater than 2. That is, if RFLEX is above 2, makespans would not depend much on actual machine assignnient.

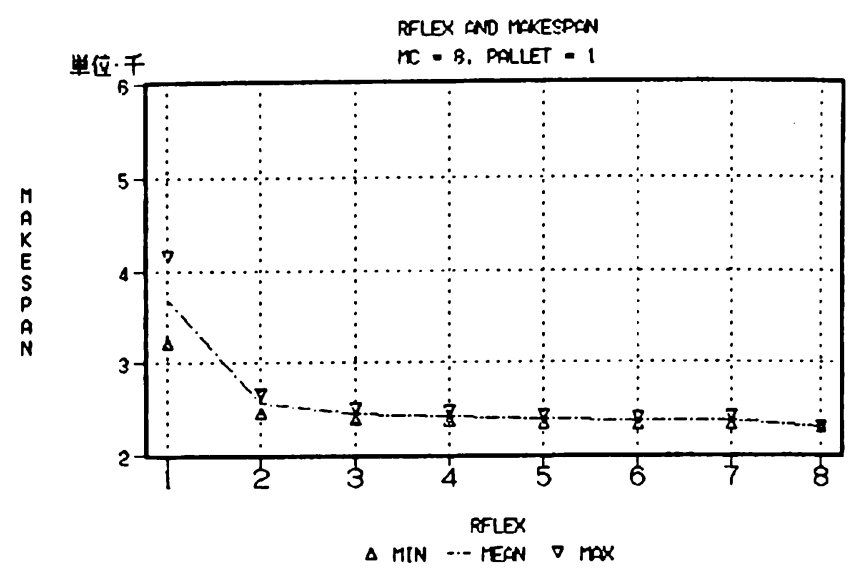

Figure 3: Effects of RFLEX

\subsection{Effects of the Number of Pallets and its Relationship to R.FLEX (Fig.4)}

In these experiments, the number of pallets are uniformly changed from 1 to 10 . That is, the equal $\mathrm{nmm-}$ ber of pallets is assumed to exist for each type of operation. Fig. 4 shows that the makespan decreases slightly as the number of pallets is increased, but, this trend does not continne long, and instead the makespan starts to increase if the number of pallets is further increased. This is probably because the system under the current configuration and also under the current control logic, gets crowded and thus increasing the waiting time of parts, when the number of pallets are increased beyond a certain level.

The effect of the number of pallets shows different characteristics depending on the value of RFIEX. That is, the increase of makespan starts even from smaller numbers of pallets, if RFLEX is kept small. The number of pallets which yields the shortest, makespan can be seen clearly. Therefore, the makespan depends much on the number of pallets.

On the other hand, as RFLEX gets bigger, the increase of makespan is not as remarkable for the smaller number of pallets, nor the number of pallets yielding the shortest makespan can be clearly identified. More specifically, makespans do not change much while the number of pallets is changed roughly between 2 and 5 . This implies that the makespan does not depend much on the number of pallets for larger values of RFLEX. 


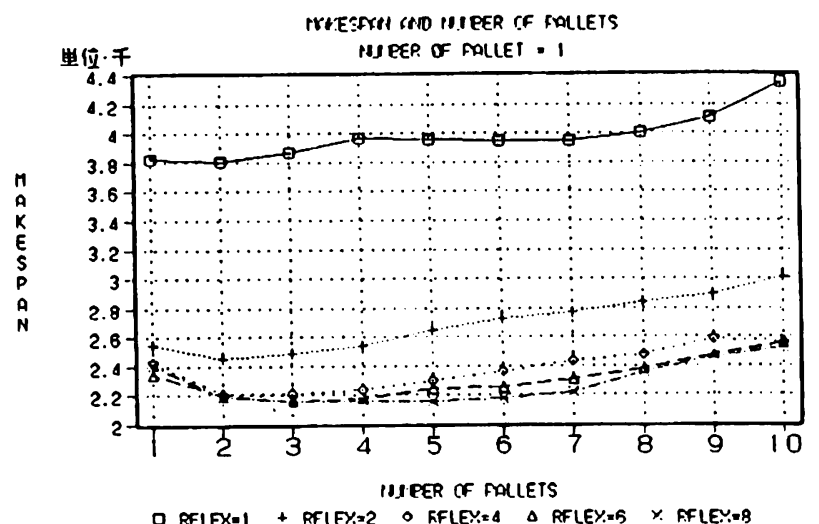

Figure 4: Effects of the Number of Pallets

The above observations indicate that increase of RFLEX has the effects of expanding the "acceptable range" of the number of entities in the system, that is, of the number of pallets. It is also obvious that graphs like Fig.4 allows one to estimate the "opti"mal" number of pallets for a particular system with a known value of RFLEX.

\subsection{Effects of the Number of Machines and its Relationship to RFLEX (Fig.5 and Fig.6)}

Withont regard to the value of RFLEX, makespan gets reduced naturally as the number of machines is increased. The rate of improvement is not uniform, and one can get bigger improvement of makespan due to the increase of the number of machines when the number of machines is smaller. Their effects are essentially the same when RFLEX is at least 2 . That is, if RFLEX is greater than or equal to 2, RFLEX cannot be regarded as a factor to reduce makespan. If RFLEX is at least 2, increase of RFLEX (above 2) cannot absorb effects of increasing the number of machines.

To be more specific, note that the makespan of the 3-machine shop with RFLEX $=2$ is approximately same as that of the 5-machine shop with RFIEX = 1. This implies that, under the condition of this simulation, increasing RFLEX from 1 to 2 has basically the same effect as increasing the number of machines from 3 to 5 . Similarly, increasing RFLEX from 1 to 2 has essentially the same effect as increasing the number of machines from 4 to 6 . In any case, it wonld be clear that the number of machines can be reduced mucl, when RFLEX is increased from 1 to 2, provided that we desire to achieve essentially the same makespan.

Fig.6 shows that the effects of changing RFIFXX would be negligibly small for RFLEX greater than or equal to 3. Therefore, the effect of increasing the number of machines cannot be achieved by increasing RFLEX.

\subsection{Effects of Allocating Machines to Opera- tions (Tables 1 - 4)}

Results described thus far indicate that the existence of several alternative machines improves the shop performance as measured by makespan. More specifically, existence of 2 or 3 alternative machines would be enough to make the system "flexible". 'The fact, that the variability of makespans is extremely small when RFIEEX goes above 2 seems to indicate that, without regard to scheduling methods employed, the makespans achieved would not differ much. That, is, makespans are relatively insensitive to scheduling of jobs.

It can also be conjectured from the large variability of makespans when RFLEX is 1 , that the allocation of particular machines to operations would greatly affect the performance of makespan. As RFLEX is increased above 2, makespans are insensitive to allocations of machines to operations. Thus, if RFLEX is at least 3 , it would not, be necessary to worry much about the machine allocation.

Based on the above observations obtained from earlier results, we analyze, in this sub-section, the effects on makespans of allocating machines to operations, and also study the effects of scheduling priority on makespans.

The hypothesis that, sched uling priority does not affect makespans is statistically confirmed at $99 \%$ confidence level, without regard to the valnes of RFIEX, allocations of machines to operations, and the number of machines.

The hypothesis which says that, the assignment, of RFLEX machines to each operation does affect makespans is statistically adopted at the confidence level of $99 \%$ when RFIEX is 1, whereas the liypothesis is not adopted for RFLEX above 2. When RFLEX is 2 , the hypothesis is/is not, adopted depending on the significance level. It is adopted at $95 \%$ significance level, but is rejected at confidence level of 99 $\%$. 


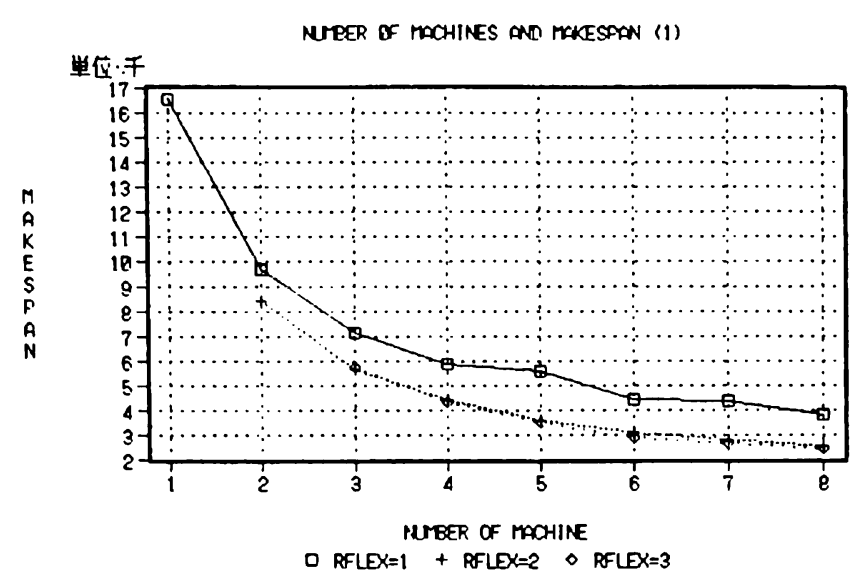

Figure 5: Effects of the Number of Machines(1)

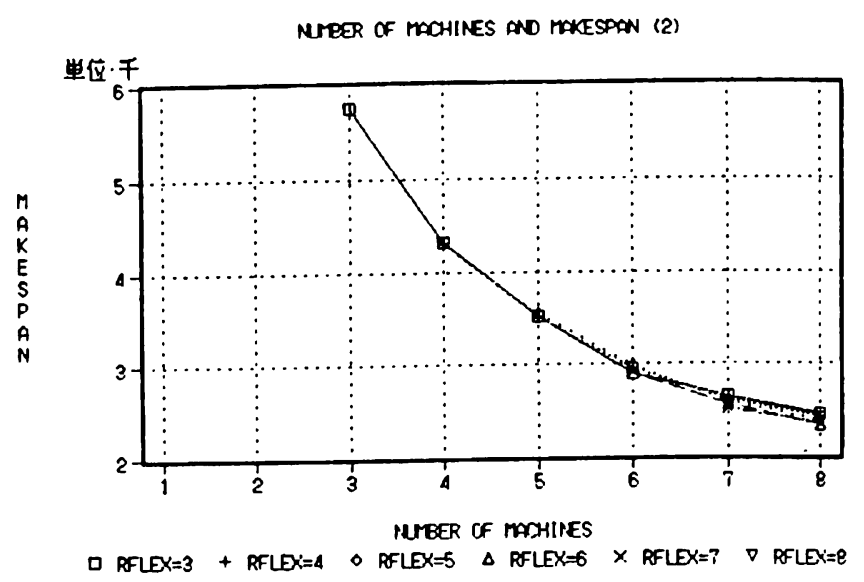

Figure 6: Effects of the Number of Machines(2)

Table 1: ANOVA Table (RFLEX $=1$ )

\begin{tabular}{c|c|c|c|c}
\hline Source & Sum of squares & Degrees of freedom & $V$ & $V / V_{e}$ \\
\hline Assignment of RFLEX & 28029696 & 9 & 3114410 & $670^{* *}$ \\
Scheduling Priority & 66304 & 9 & 7367 & 1.58 \\
Error & 376064 & 81 & 4642 & \\
Total & 28472064 & 99 & & \\
\hline
\end{tabular}

Table 2: ANOVA Table (RFLEX $=2)$

\begin{tabular}{c|c|c|c|c}
\hline Source & Sum of squares & Degrees of freedom & $V$ & $V / V_{e}$ \\
\hline Assignment of RFLEX & 176256 & 9 & 19584 & $2.30^{*}$ \\
Scheduling Priority & 61824 & 9 & 6869 & 0.807 \\
Error & 689344 & 81 & 8510 & \\
Total & 927424 & 99 & & \\
\hline
\end{tabular}

Table 3: ANOVA Table (RFLEX = 3)

\begin{tabular}{c|c|c|c|c}
\hline Source & Sum of squares & Degrees of freedom & $V$ & $V / V_{e}$ \\
\hline Assignment of RFLEX & 85248 & 9 & 9472 & 1.66 \\
Scheduling Priority & 38656 & 9 & 4295.11 & 0.752 \\
Error & 462656 & 81 & 5711.8 & \\
e Total & 5865650 & 99 & & \\
\hline
\end{tabular}

Table 4: ANOVA Table (RFLEX $=5$ )

\begin{tabular}{c|c|c|c|c}
\hline Source & Sum of squarcs & Degrees of freedom & $V$ & $V / V_{e}$ \\
\hline Assignment of RFLEX & 53140 & 9 & 5937.78 & 1.38 \\
Scheduling Priority & 18240 & 9 & 2026.67 & 0.470 \\
Error & 349312 & 81 & 4312.49 & \\
Total & 420992 & 99 & & \\
\hline
\end{tabular}

$F_{81}^{9}(0.05)=2.00 \quad F_{81}^{9}(0.01)=2.64$

* significant at the $5 \%$ level ** significant, at the $1 \%$ level 


\section{CONCLUSIONS}

Analysis of commercial custom-made FMSs with discrete event simulation is described with special emphasis on the flexibility of the system achived by providing alternative machines that can perform an operation. It was found that having just 2 or 3 alternative machines from which one would eventually be selected for actual machining by the FMS control logic, would greatly increase the flexibility of the system. Contributions of having more than 3 alternative machines would be very small.

It is found that candidate machine assignment and scheduling priority would not affect FMS performance as measured by makespan, provided that a reasonable number (say, 2 or 3 ) of alternative machines are provided so that parts could take several alternative routes. It is also found that scheduling priority does not affect makespan performance. Careful machine assignment, on the other hand, would be necessary to achieve short makespan, if RFLEX is small (say, 1).

\section{ACKNOWLEDGMENTS}

This research is supported by Waseda University Grant for Special Research Projects (90^-84, 91^-79, 91C-7), a research grant from Progressive Manufacturing Foundation and Ministry of Education, Grantin-Aid for General Scientific Research (C 132-268).

The anthors would like to express appreciations to Mr.Hiroshi Awane and Mr.Makoto Tanahashi of Yamazaki Mazak for suggestions, discussions, and also supply of data.

\section{REFERENCES}

Carrie, A. 1988. Simulation of Manufacturing Systems. Chichester: John Wiley \& Sons.

Sethi, A.K. and S.P.Sethi. 1990. Flexibility in manufacturing: a survey. International Journal of Flexible Manufacturing Systems 2: 289-328.

Talavage, J. and R.G.Hannam. 1988. Flexible Manufacturing Systems in Practice: Applications, Design, and Simulation. New York: Marcel Dekker.

\section{AUTHOR BIOGRAPHIES}

SUSUMU MORITO is Professor of Operations Research at Department of Industrial Engineering and Management, School of Science and Engineering, Waseda University. He currently serves as Associate
Dean of the Graduate School of Science and Fngineering at Waseda. He received his B.Engr. and M.Engr. from Waseda, and also M.S. and Ph.D. from Department of Operations Research, Case Western Reserve University, Cleveland, Olio. Professor Morito t,aught, at Case Western Reserve and also at the University of Tsukuba prior to joining Waseda. His main area of research is operations research, with particular emphasis on applications of discrete-event simulation in manufacturing. He serves as the president of the special interest group on simulation at the Operations Research Society of Japan.

TOMOHIRO TAKANO just started to work at Industrial Engineering Production System Design Department, Brigestone Corporation, after receiving M. Engr. in Industrial Engineering from Waseda University. He also holds B. Engr. from Waseda.

HISANOBU MIZUKAWA is Graduate Student working for his master's thesis at Department of Industrial Engineering and Management, Waseda University, where he received B. Engr.

KIYOHIS A MIZOGUCHI is Manager of Engineering Department at YAMAZAKI MAZAK CORPORATION. 